Rita de Cássia Pereira Fernandes 1

\title{
Trabalho e cárcere: um estudo com agentes penitenciários da Região Metropolitana de Salvador, Brasil
}

Annibal Muniz Silvany Neto 2

Gildélia de Miranda Sena 1

Alexandre dos Santos Leal 2

Carina Amorim Pouillard Carneiro 2

Fernanda Pita Mendes da Costa 2

\author{
Work in the prison system: a study of correctional \\ officers in Greater Metropolitan Salvador, Brazil
}

1 Centro de Estudos da Saúde do Trabalhador, Secretaria de Saúde do Estado da Bahia. Rua Pedro Lessa 123.

Salvador, BA

40110-050, Brasil.

2 Departamento de Medicina

Preventiva, Faculdade de

Medicina, Universidade

Federal da Bahia.

Av. Reitor Miguel Calmon $s / n$,

Campus Universitário

do Canela, Salvador, BA 40110-100, Brasil.

\begin{abstract}
A cross-sectional study was conducted to identify possible associations between working conditions and health among correctional officers in Salvador, Bahia, Brazil. The study used a stratified proportional random sample of 311 individuals who answered a non-identifiable self-applied questionnaire. The following results were obtained by logistic regression: (a) psychologically inadequate workplace, insufficient basic work conditions, no leisure time, lack of sports practice, $\geq$ nine years working in the prison system, excessive work hours, $\geq 48$ hours work/week, and inadequate organizational conditions at work were associated with minor psychological disorders; (b) lack of specific training, femal e gender, $\geq 48$ hours work/week, psychol ogi cally inadequate workplace, no leisure time, and lack of sports practice were associated with persistent stress, (c) age $\geq 45$ years, $\geq$ nine years working in the prison system, excessive work hours, lack of sports practice, inadequate basic and organizational work conditions, and minor psychological di sorders were associated with heal th complaints.
\end{abstract}

Key words Prisons; Occupational Health; Mental Health; Cross-Sectional Studies; Psychopathology

Resumo Estudo transversal para identificar possíveis associações entre condições de trabal ho e saú de de agentes penitenciários de Salvador, Bahia, Brasil, utilizou uma amostra aleatória estratificada proporcional de 311 indivíduos, que responderam, sem identificação, questionário auto-aplicável. Obteve-se os seguintes resultados na regressão logística: (a) ambiente de trabal ho psi col ogi camente inadequado, condi ções infra-estruturais insuficientes, falta de tempo para lazer, ausência de esporte, mais de nove anos no Sistema Penitenciário (SP), dobra de turno, jornada $>48$ horas semanai s e organização do trabal ho inadequada, foram associados positivamente com distúrbios psíqui cos menores (DPM); (b) falta de trei namento, sexo femi ni no, jornada $>48$ horas semanais, ambi ente de trabal ho psicologicamente inadequado, falta de tempo para lazer e ausência de esporte, foram associados positivamente com estresse persistente; (c) idade $\leq 45$ anos, $\geq$ nove anos no SP, dobra de turno, ausência de esporte, ambiente de trabal ho psi col ogi camente inadequado, condições infra-estruturais e organizacionais inadequadas e presença de DPM, foram associados positivamente com queixas de saú de

Palavras-chave Prisões; Saúde Ocupacional; Saúde Mental; Estudos Tranversais; Psicopatologia 


\section{Introdução}

O Sistema Penitenciário (SP) no Brasil e na Bahia passa por uma crise como indicam as celas superlotadas, conflitos entre quadrilhas e o tráfico de drogas dentro das suas Unidades, com elevado número de rebeliões caracterizadas por grande violência.

Os Agentes Penitenciários (AP) são os trabalhadores encarregados de revistar presos, celas, visitantes, conduzir presos, realizar a vigilância interna da Unidade e disciplinar a refeição dos presos. Por terem contato direto com os internos e sendo vistos por estes como um dos responsáveis pela manutenção do seu confinamento, estes trabalhadores estão freqüentemente expostos a diversas situações geradoras de estresse, tais como intimidações, agressões e ameaças, possibilidade de rebeliões nas quais, entre outros, correm o risco de serem mortos ou se tornarem reféns.

Policiais e AP foram referidos por Tartaglini \& Safran (1997), como profissionais submetidos a um alto risco para a doença relatada como estresse debilitante. Estes autores encontraram prevalências de ansiedade, distúrbios de comportamento e abuso de álcool mais altos entre os AP do que na população em geral. Relataram entre esses trabalhadores, uma prevalência de distúrbios emocionais de 18,6\%, abuso de álcool de 4,5\% e distúrbios da ansiedade de 7,9\%.

Em estudo realizado na França, com todas as categorias de trabalhadores de prisão, Goldberg et al. (1996) observaram prevalências de $24 \%$ de sintomatologia depressiva, $24,6 \%$ de distúrbios da ansiedade e $41,8 \%$ de distúrbios do sono.

Um estudo realizado em Nova Iorque, por Steenland et al. (1997) no período de 1991 a 1993, encontrou que os AP representavam um grupo de risco importante para infecção pelo bacilo da tuberculose. Aproximadamente, 33\% dos novos casos de tuberculose entre os agentes, foram considerados ocupacionais por esses autores.

A maior parte dos estudos revisados investigava as condições de saúde dos presos. Muito pouco foi estudado sobre a saúde dos AP. O presente estudo teve o objetivo de investigar, exploratoriamente, possíveis relações entre condições de trabalho e saúde em AP das oito Unidades do SP da Região Metropolitana de Salvador (RMS). Os resultados obtidos poderão fundamentar algumas mudanças nas condições de trabalho, com possível influência sobre a saúde desses trabalhadores, e/ ou gerar hipóteses a serem testadas em novas pesquisas.

\section{Metodologia}

Foi realizado um estudo epidemiológico transversal. A população-alvo abrangeu todos os AP da RMS.

$\mathrm{Na}$ fase de planejamento do estudo, foi realizada uma visita técnica às instalações de uma das Unidades Carcerárias que seriam estudadas, para se conhecer com maiores detalhes o processo de trabal ho e a dinâmica própria da categoria de trabalhadores a ser estudada. Essas informações foram utilizadas na definição e aperfeiçoamento do questionário utilizado no estudo e nos procedimentos de coleta de dados.

Do total de 628 agentes, estudou-se uma amostra de 311 indivíduos selecionados por amostragem aleatória estratificada proporcional. A amostra manteve a mesma proporcionalidade entre o número de agentes de cada unidade penitenciária e aquela existente na população-alvo. O tamanho mínimo para a amostra foi calculado considerando-se um grau de precisão absoluta de 5,0\%, nível de confiança de 95,0\%, prevalência esperada de 50,0\% e um efeito de desenho de 1.3, conforme procedimento recomendado por Daniel (1987). Agentes de todas as Unidades do SP da RMS estavam representados na amostra.

Apenas os trabalhadores oficialmente classificados como AP pela Secretaria de Justiça foram considerados para estudo. AP sorteados $\mathrm{e}$ que se encontravam nas condições abaixo, foram substituídos mediante sorteio, desde que o motivo do afastamento (licença maternidade, convalescença de cirurgia estética, seqüela de acidente automobilístico não relacionado com o trabalho) não tivesse relação presumível com as condições investigadas: agentes afastados há um ano e que trabalharam como AP por menos de cinco anos; afastados há dois anos e com menos de dez de trabal ho como AP e afastados há mais de dois anos, independentemente do tempo de trabalho como AP.

Para a coleta de informações, foi utilizado um questionário auto-aplicável, padronizado. Esse questionário continha perguntas que visavam a coleta de informações sobre condições sócio-demográficas, aspectos ocupacionais, hábitos de vida, morbidade geral e ocupacional, presença de distúrbios psíquicos menores (DPM), consumo excessivo de bebidas alcoólicas e existência de estresse.

Para coleta das informações relativas à DPM , foi utilizado o SRQ-20 (Self Report Questionnaire). Esse questionário foi projetado por Harding et al. (1980, apud Mari, 1986) para uso em estudos de morbidade psiquiátrica em ins- 
tituições de cuidados primários de saúde, em países em desenvolvimento coordenados pela Organização Mundial da Saúde (OMS). O SRQ é derivado de quatro instrumentos de pesquisa psiquiátrica já existentes. É um instrumento auto-aplicável contendo uma escala de respostas sim/não. A versão em português adotou os vinte primeiros itens para morbidade não psicótica. Foi adotado o ponto de corte em sete ou mais respostas positivas, classificando-se estes indivíduos como portadores de DPM. Esse escore foi validado por Fernandes (1993), Harding et al. (1980, apud Mari, 1986) e por Mari (1986) que encontraram, respectivamente, sensibilidades e especifidades de 57,1 e 79\%, 73 a $83 \%$ e 72 a $85 \%$, 83 e $80 \%$.

Para avaliação de consumo excessivo de bebidas alcoólicas (suspeita de alcoolismo) utilizou-se o CAGE, que é também auto-aplicável e composto por quatro questões. O termo CAGE é originário das palavras-chave incluídas em cada uma das suas questões em inglês, respectivamente: Cut-down, Annoyed, Guilty, Eye-opener. A validação do CAGE, no Brasil, foi feita por Masur \& Monteiro (1983). Adotando um ponto de corte em duas ou mais respostas positivas, a validação mostrou uma sensibilidade de $88 \%$ e uma especificidade de $83 \%$. Esse foi o escore de corte usado no presente estudo.

O Inventário de Sintomas de Stress (ISS), elaborado por Lipp et al. (1994), visa identificar de modo rápido e objetivo a sintomatologia que o paciente apresenta, avaliando se o indivíduo possui sintomas de estresse, o tipo de sintoma existente (somático ou psicológico) e a fase de estresse em que se encontra. No presente estudo, utilizou-se uma versão resumida do ISS, sugerida por Lipp (Bernardes, 1997). Esse questionário é composto de três partes e cada parte corresponde a uma fase do estresse. Na primeira parte, avaliam-se os sintomas experimentados nas últimas $24 \mathrm{~h}$. Nessa fase de alerta, o organismo se prepara para a reação de luta ou fuga. Na segunda parte, avaliam-se os sintomas experimentados na última semana. Nessa fase, as reações são opostas àquelas da primeira e muitos dos sintomas iniciais desaparecem, dando lugar a uma sensação de desgaste e resistência. Se o estresse é contínuo e a pessoa não possui estratégias para lidar com este, ela entra na fase de exaustão, quando doenças sérias aparecem. Essa fase corresponde à terceira parte do questionário, em que se avaliam os sintomas experimentados no último mês. O escore de corte para cada uma dessas partes foi de cinco ou mais respostas positivas às questões formuladas, classificando-se os tipos de estresse correspondentes a cada parte como mo- mentâneo, intermediário ou persistente, respectivamente.

As questões relativas às condições de trabaIho foram respondidas pelo agente em uma escala variando de 0 a 5, representando o grau de avaliação que este fazia acerca de sua atividade. Com esse procedimento e com a formulação adequada das questões, buscou-se evitar a indução do agente penitenciário a uma resposta favorável ou desfavorável sobre cada condição estudada.

Foi realizado um pré-teste do questionário, visando seu aperfeiçoamento e o estabelecimento de condutas mais adequadas na realização da coleta de dados, que foi realizada por estudantes de medicina da Universidade Federal da Bahia (UFBA) e equipe técnica da pesquisa, sendo suas condutas padronizadas. Os questionários foram aplicados nas próprias unidades penitenciárias. Se o AP não fosse encontrado na primeira tentativa, novas visitas eram feitas até a aplicação do questionário, sendo a entrega e recebimento deste feitos diretamente pelos membros da equipe de pesquisa. Não foi permitido que os agentes permanecessem com o questionário em mãos para entrega posterior. A coleta de dados foi realizada entre 5 de outubro e 31 de dezembro de 1999.

A análise estatística dos dados constou de uma etapa descritiva, seguida de análise tabular, com realização de testes do qui-quadrado, cálculo de Razões de Prevalência (RP) e de seus respectivos intervalos de confiança, obtidos pelo método de Cornfield. A análise tabular bivariada foi conduzida separadamente para quatro variáveis dependentes: DPM, suspeita de alcoolismo, queixas gerais de saúde e estresse persistente. Os resultados da análise bivariada não são relatados, mas serviram para um melhor conhecimento do banco de dados e foram utilizados para orientar as etapas mais avançadas de análise estatística.

Consi derando-se que, as trinta e uma variáveis relativas às condições de trabalho eram muito relacionadas entre si, fez-se uma redução de variáveis através de uma análise de fator (Kleinbaum et al., 1988). A extração inicial foi feita por meio de uma análise de componentes principais, e a rotação realizada foi ortogonal (varimax). A análise multivariável, também exploratória, foi conduzida por Regressão Logística (RL), separadamente, para as quatro variáveis dependentes estudadas. A RL foi iniciada com uma pré-seleção de variáveis, baseada na plausibilidade biológica das associações envolvidas, e em regressões logísticas univariadas, tendo sido pré-selecionadas aquelas 
variáveis com um valor de $\mathrm{P}$ menor ou igual a 0,25 no teste da razão de verossimilhança. 0 método de seleção de variáveis foi o de trás para a frente "backward", com reavaliação a cada etapa "stepwi se". O $\alpha$ utilizado nesta etapa foi de 0,17 para remoção e de 0,16 para entrada no modelo (Hosmer \& Lemeshow, 1989). O valor de $P$ para remoção, foi aquele obtido no teste da razão de verossimilhança e, para entrada, no teste do escore. No diagnóstico da regressão, utilizaram-se o teste de qualidade do ajuste de Hosmer \& Lemeshow, e a análise de resíduos para identificação e avaliação de indivíduos com resíduos estudantizados, distância de Cook, DFBetas e desvios "deviance" anômalos (acima de dois desvios-padrão), (Hosmer \& Lemeshow, 1989). Considerando-se que, os problemas de saúde estudados apresentaram freqüência elevada (maior do que 5,0\%), as razões de chance obtidas foram transformadas em razões de prevalência, utilizando-se o método delta (Oliveira et al., 1997).

Os intervalos de confiança foram calculados sem considerar o efeito de desenho.

$\mathrm{Na}$ etapa de interpretação dos resultados, estes foram discutidos com agentes penitenciários, entre eles os chefes de segurança das unidades, para obter-se um conhecimento mais detalhado sobre que processos específicos do seu trabalho poderiam explicar esses achados.

Foi garantido aos participantes o sigilo da sua identidade em todas as etapas desta pesquisa.

\section{Resultados}

Foram estudados 311 agentes penitenciários das oito Unidades Penitenciárias da Região Metropolitana de Salvador. Os homens representaram 81,3\% da amostra estudada. Cerca de $47 \%$ dos agentes eram casados, $73,0 \%$ tinham o segundo grau completo, $18,0 \%$ o nível superior de escolaridade e 70,4\% eram naturais de Salvador.

Cerca de $44,0 \%$ realizaram treinamento para a função. Mais de um terço dos AP realizavam outra atividade remunerada. Destes, $27,6 \%$ afirmaram que essa atividade extra era feita na área de segurança. Aproximadamente, 13,0\% dos AP costumavam dobrar o turno na penitenciária. Observou-se que, 89,0\% trabalhavam no regime de turno, cumprindo plantões de $24 \mathrm{~h}$, seguidas de $72 \mathrm{~h}$ de folga e $11,0 \%$ trabal havam no regime administrativo, desenvolvendo atividades atípicas para a função, como as de suporte administrativo.

O uso de drogas ilícitas não foi relatado por nenhum deles. Mais de três quartos (76,45\%) afirmaram dispor de tempo para o lazer. Apenas $15,8 \%$ relataram a disponibilidade de equipamentos de proteção individual no trabal ho da penitenciária.

Quanto ao consumo de álcool, aproximadamente dois terços $(68,5 \%)$ afirmaram que bebiam atualmente e apenas $14,8 \%$ relataram nunca ter consumido.

A média de idade foi de $40,2 \pm 7,7$ anos, o tempo médio de função como AP foi de 10,3 \pm 5,8 anos e de $7,5 \pm 6,2$, o tempo médio na unidade penitenciária; a média de horas semanais de trabalho na prisão foi de $47,1 \pm 6,7$ horas e a média de horas semanais de trabalho em outras atividades remuneradas foi de $38,0 \pm 16,3 \mathrm{~h}$.

A prevalência de DPM foi $30,7 \%$, de estresse passageiro 7,4\%, estresse intermediário 7,4\% e estresse persistente 15,1\%. Queixas de doenças foram feitas por $91,6 \%$ dos AP (53,1\% apresentaram até 5 queixas e $38,5 \%$, mais de 5$)$. Houve suspeita de alcoolismo em 15,6\% (Tabela 1).

A análise de fator resultou em cinco fatores, cuja composição, em ordem decrescente das cargas apresentadas por cada variável, pode ser vista a seguir: Fator 1 (condições do alojamento, condições do refeitório, qualidade da alimentação, adequação de mobiliário, luminosidade, ventilação, qualidade da água, tempo para refeições e disponibilidade de materiais); Fator 2 (satisfação no desempenho das atividades, relação com a chefia, independência na realização das atividades, relação com colegas, tranqüilidade, monotonia e supervisão); Fator 3 (repetitividade de tarefas, barulho, umidade, pressão da direção e tamanho da equipe; Fator 4 (dificuldade de transporte, variedade de funções, ritmo de trabalho e obstáculos para execução das atividades); Fator 5 (trabalho em pé, sentado, subindo e descendo escadas e comodidade da posição de trabalho).

As Tabelas 2, 3, 4 e 5 apresentam os resultados da análise multivariável. O teste de qualidade e as análises dos resíduos mostraram um bom ajuste dos modelos.

\section{Discussão e conclusões}

Há mais homens entre os AP. Isso deve ocorrer devido à existência de uma proporção também maior de homens infratores, pois um presidiário só pode ser vigiado por um agente do mesmo sexo.

A escolaridade deste grupo ocupacional é elevado. Mais recentemente, tem sido exigida conclusão do nível médio nos concursos realizados. Foram encontrados vários AP com nível superior de escolaridade. Tal achado deve re- 
fletir uma dificuldade de inserção desses indivíduos no mercado de trabalho, que podem ter buscado na atividade de agente uma situação provisória. Com o aumento do desemprego, a possibilidade de mudança deve ter se tornado mais difícil, fazendo perdurar a inserção como AP.

Mais da metade dos agentes afirmou não ter sido treinada para a função. Diante da complexidade das suas atividades, o agente necessita de um grande preparo para lidar com indivíduos infratores que, isoladamente ou em grupo, tentam questionar constantemente sua autoridade. Além disso, trata-se de um ambiente de trabalho bastante especial do ponto de vista das relações interpessoais e da autoridade exigi da do AP. Um treinamento adequado seria indispensável para que o trabalhador pudesse exercer sua autoridade sem utilizar o recurso mais fácil da violência constante, ou de se deixar ceder ou corromper diante de ameaças e propostas feitas.

Muitos AP trabalham nos dias de folga, possivelmente para aumentar sua renda. Além disso, vários agentes costumam dobrar o turno, o que também pode indicar um esforço para meIhorar o salário. E é possível ainda, que vários AP tenham omitido a informação sobre outro trabalho remunerado, com receio de que sua atividade nos horários de folga, pudesse ser vista como fonte de sobrecarga que poderia repercutir negativamente sobre seu desempenho no SP.

Entre os AP com outra atividade remunerada, esperava-se uma proporção maior em atividade de segurança. Essa possível subnotifi- cação poderia ser explicada por uma necessidade de afirmação do agente de que sua atividade no SP se diferencia daquilo que habitualmente caracteriza o trabalho de um segurança, voltado para vigilância repressiva e passível de uso constante da violência. Ou seja, ser AP não o habilitaria, necessariamente, para as funções típicas de segurança, tendo em vista que a atribuição precípua do primeiro seria reabilitar o preso para o convívio com a sociedade.

A observação de desvio de função, pode representar uma tentativa de evitar o trabal ho perigoso, fisicamente e psicologicamente desgastante, de contato direto com os presos. Outra explicação possível e compatível com a ante-

Tabela 1

Prevalência dos agravos à saúde na amostra de agentes penitenciários. Região Metropolitana de Salvador, 2000

\begin{tabular}{|c|c|c|c|}
\hline Agravo & $n(N)$ & Prevalência (\%) & IC $95 \%$ \\
\hline Distúrbios psíquicos menores & 95 (309) & 30,7 & $25,5-35,8$ \\
\hline \multicolumn{4}{|l|}{ Estresse } \\
\hline Momentâneo & $23(311)$ & 7,4 & $4,5-10,3$ \\
\hline Intermediário & $23(310)$ & 7,4 & $4,5-10,3$ \\
\hline Persistente & 45 (309) & 15,1 & $11,1-19,1$ \\
\hline Queixas & 283 (309) & 91,6 & $88,5-94,7$ \\
\hline \multicolumn{4}{|l|}{ Número de queixas } \\
\hline Sem queixas & $26(311)$ & 8,4 & $5,3-11,5$ \\
\hline Até 5 queixas & $164(311)$ & 53,1 & $47,5-58,6$ \\
\hline Mais de 5 queixas & $119(311)$ & 38,5 & $33,1-43,9$ \\
\hline Suspeita de alcoolismo (CAGE) & $34(218)$ & 15,6 & $10,8-20,4$ \\
\hline
\end{tabular}

Tabela 2

Resultados do modelo final da análise multivariável: distúrbios psíquicos menores como variável dependente. Amostra de agentes penitenciários $(n=245)$, Região Metropolitana de Salvador, 2000.

\begin{tabular}{lrrrr}
\hline Variável independente & $\beta$ & Erro-padrão & RC (IC 95\%) & RP (IC 95\%) \\
\hline Fator 2 & 1,19 & 0,35 & $3,28(1,65-6,49)$ & $1,97(1,34-2,91)$ \\
Ausência de prática de esporte & 1,03 & 0,33 & $2,79(1,46-5,33)$ & $1,76(1,15-2,68)$ \\
Fator 4 & 0,97 & 0,37 & $2,62(1,27-5,42)$ & $1,75(1,16-2,65)$ \\
Costume de dobrar o turno & 0,65 & 0,44 & $1,91(0,80-4,54)$ & $1,64(1,02-2,63)$ \\
Tempo de trabalho em anos $(\geq 9 ;<9)$ & 0,79 & 0,39 & $2,21(1,03-4,73)$ & $1,58(0,92-2,73)$ \\
Ausência de tempo para lazer & 0,81 & 0,37 & $2,26(1,08-4,69)$ & $1,54(0,99-2,38)$ \\
Fator 1 & 0,75 & 0,41 & $2,10(0,95-4,68)$ & $1,43(0,83-2,45)$ \\
Fator 3 & 0,44 & 0,32 & $1,56(0,83-2,92)$ & $1,39(0,91-2,12)$ \\
Total de horas semanais de trabalho $(>48 ; \leq 48)$ & 0,49 & 0,35 & $1,56(0,83-2,92)$ & $1,27(0,82-1,97)$ \\
Constante & $-3,68$ & 0,60 & - & - \\
\hline
\end{tabular}

Teste de qualidade do ajuste: $\chi^{2}=9,4005 ;$ g.l. $=8 ; p=0,3096$. 
Tabela 3

Resultados do modelo final da análise multivariável: estresse persistente como variável dependente. Amostra de agentes penitenciários $(n=264)$, Região Metropolitana de Salvador, 2000

\begin{tabular}{lcccc}
\hline Variável independente & $\beta$ & Erro-padrão & RC (IC 95\%) & RP (IC 95\%) \\
\hline Total de horas semanais de trabalho $(>48 ; \leq 48)$ & 1,13 & 0,43 & $3,09(1,34-7,15)$ & $2,95(1,42-6,16)$ \\
Ausência de treinamento & 1,27 & 0,44 & $3,57(1,50-8,47)$ & $2,87(1,32-6,24)$ \\
Fator 2 & 1,20 & 0,43 & $3,34(1,43-7,75)$ & $2,76(1,31-5,80)$ \\
Sexo Feminino & 0,98 & 0,43 & $2,68(1,15-6,22)$ & $2,64(1,28-5,44)$ \\
Ausência de prática de esporte & 0,85 & 0,40 & $2,35(1,06-5,20)$ & $2,46(1,19-5,08)$ \\
Ausência de tempo para lazer & 0,68 & 0,44 & $1,98(0,83-4,69)$ & $1,75(0,83-3,72)$ \\
Constante & $-4,58$ & 0,61 & - & - \\
\hline
\end{tabular}

Teste de qualidade do ajuste: $\chi^{2}=4,4078 ;$ g.l. $=8 ; p=0,8182$.

Tabela 4

Resultados do modelo final da análise multivariável: suspeita de alcoolismo como variável dependente. Amostra de agentes penitenciários $(n=243)$, Região Metropolitana de Salvador, 2000.

\begin{tabular}{lcccr}
\hline Variável independente & $\beta$ & Erro-padrão & RC (IC 95\%) & RP (IC 95\%) \\
\hline Tabagismo & 1,27 & 0,48 & $3,56(1,40-9,07)$ & $2,22(1,10-4,46)$ \\
Outra atividade remunerada & 1,27 & 0,47 & $3,57(1,42-8,99)$ & $2,09(1,05-4,18)$ \\
Ausência de treinamento & 0,92 & 0,49 & $2,51(0,96-6,56)$ & $1,66(0,81-3,11)$ \\
Ausência de tempo para lazer & 0,80 & 0,47 & $2,22(0,88-5,55)$ & $1,65(0,80-3,38)$ \\
Constante & $-3,97$ & 0,57 & - & - \\
\hline
\end{tabular}

Teste de qualidade do ajuste: $\chi^{2}=3,3932 ;$ g.l. $=5 ; p=0,6396$.

Tabela 5

Resultados do modelo final da análise multivariável: queixas gerais de saúde como variável dependente. Amostra de agentes penitenciários ( $n=236$ ), Região Metropolitana de Salvador, 2000.

\begin{tabular}{lcccc}
\hline Variável independente & $\beta$ & Erro-padrão & RC (IC 95\%) & RP (IC 95\%) \\
\hline DPM & 1,95 & 0,35 & $7,03(3,55-13,95)$ & $3,12(2,24-4,45)$ \\
Fator 3 & 0,90 & 0,33 & $2,45(1,28-4,72)$ & $1,99(1,31-3,02)$ \\
Costume de dobrar o turno & 1,24 & 0,51 & $3,45(1,29-9,21)$ & $1,83(1,17-2,85)$ \\
Tempo de trabalho em anos $(\geq 9 ;<9)$ & 0,75 & 0,40 & $2,12(9,97-4,64)$ & $1,78(1,00-3,17)$ \\
Fator 1 & 0,72 & 0,34 & $2,05(1,06-3,97)$ & $1,66(1,10-2,52)$ \\
Fator 2 & 0,50 & 0,34 & $1,66(0,87-3,20)$ & $1,45(0,97-2,19)$ \\
Ausência de prática de esporte & 0,65 & 0,34 & $1,92(0,99-3,72)$ & $1,38(0,93-2,07)$ \\
Idade $>45 ; \leq 45)$ & $-0,72$ & 0,44 & $0,49(0,20-1,17)$ & $0,58(0,31-1,08)$ \\
Constante & $-3,12$ & 0,54 & - & - \\
\hline
\end{tabular}

Teste de qualidade do ajuste: $\chi^{2}=4,4798 ;$ g.l. $=8 ; p=0,8114$. 
rior, seria a existência de uma carência de pessoal nos setores administrativos das Unidades, que vem sendo minimizada às custas da redução do efetivo dos AP das funções típicas, e sua transferência para o suporte administrativo nas Unidades. O desvio de função traz implicações negativas para as condições de trabalho daqueles que permanecem na atividade junto ao cárcere, agravando sua carga de trabalho (Wisner, 1994).

Nenhum AP referiu o uso de drogas ilícitas. Apesar da garantia de anonimato, este resultado deve estar subestimado, considerando-se o contato dos AP com traficantes e usuários de drogas por força da profissão, e a potencial vulnerabilidade do AP dentro das estruturas informais de poder que se estabelecem no cotidiano das Unidades Carcerárias. Esse achado é discordante do estudo de Svenson et al. (1995) no Canadá, no qual 58,0\% dos AP relataram o uso, no passado, de alguma droga ilícita e 20,0\% indicaram uso corrente. Essas proporções foram muitas vezes maiores do que o uso referido pela população geral do mesmo país. Esses padrões de uso encontrados entre os AP, são mais próximos daqueles observados entre os encarcerados.

O tempo médio no SP (10,3 anos) reflete o tipo de vínculo dessa categoria, com estabilidade no emprego. Essa média de tempo parece estar bem acima daquilo que éa experiência de outras categorias de trabal hadores (Araújo et al., 1998).

Comparando-se o tempo médio de trabaIho no SP e na Unidade de lotação no momento da coleta de dados, a diferença não era muito acentuada, o que deve indicar uma baixa rotatividade dos AP. A pequena mobilidade existente ocorre, em geral, por transferência do AP devido a problemas disciplinares mais graves (uso e tráfico de drogas, corrupção, negligência) ou a pedido dele, caso em que ele precisará da ajuda da hierarquia mais alta do SP. Na primeira situação, a depender do local para onde o agente seja transferido (Central Médica, Casa de Albergados, por exemplo) a mudança poderá ser considerada como uma premiação ao agente transgressor, fato que tem provocado intensa insatisfação dos trabalhadores que continuam a realizar seu trabal ho perigoso em um presídio ou penitenciária.

A prevalência de DPM encontrada $(30,7 \%)$ foi mais elevada do que aquelas encontradas por Araújo et al. (1998) de 20,1\%, Borges (1990) de 19,0\%, Fernandes (1993) de 19 a 24\%, Pitta (1990) de 20,8\%, em amostras de professores da rede particular de ensino de Salvador, metalúrgicos, trabal hadores de processamentos de dados e de hospitais, respectivamente.
O número de AP com pelo menos um problema ou sintoma de doença foi muito elevado, e o número deles com mais de cinco dessas queixas foi também bastante alto. Isto representa uma morbidade referida elevada no grupo estudado, e a análise realizada sugere que esta pode estar relacionada com algumas condições de trabalho, além de estar consistente com o que tem sido referido por outros autores (Kauffman, 1988, apud Svenson et al., 1995).

Considerando os resultados da análise multivariável, observou-se que sete das variáveis independentes estatisticamente, e independentemente, associadas a DPM dizem respeito às condições de trabalho do AP. A associação encontrada com o fator 2 é consistente com a literatura, que tem apontado forte associação entre as cargas relacionadas com as relações interpessoais, relações hierárquicas, estrutura de poder na organização, intranqüilidade e o sofrimento psíquico dos trabalhadores (Dejours, 1987; Silva, 1993). As ocupações que se caracterizam pelo contato com um contingente de indivíduos, determinam o envolvimento dos trabalhadores com as manifestações de descontentamento desse grupo. Os AP enfrentam, de maneira habitual e permanente, as manifestações dos encarcerados contra o SP e esta atitude negativa dos sujeitos com os quais se trabalha implica sofrimento mental naqueles que respondem pelo SP (Wisner, 1994). Ainda segundo Wisner, 1994, embora o custo psíquico sofrido com as agressões no trabalho varie muito de acordo com as características de cada sujeito, ele sempre existirá. À angústia corresponde a ansiedade e o medo, ligados às decisões a tomar e às situações perigosas. Os AP experimentam durante toda sua jornada, uma incerteza quanto à eficiência da segurança na Unidade onde trabal ham. A possibilidade de rebelião, fuga em massa, resistência armada de presos em decorrência de falhas na segurança são preocupações diárias. Os AP não têm participado das decisões gerenciais do SP, que implicam mudanças diretas na segurança das Unidades, sendo estas potencialmente geradoras de tensões e medos. Os "ingressos", familiares que visitam e permanecem com os presos alguns dias, aumentam consideravelmente a população dentro das Unidades, o que exige uma maior vigilância por parte dos AP, tendo em vista a possibilidade de entrada de armas e drogas. Embora essas visitas especiais possam ser benéficas para o processo de re-socialização do preso, sua implementação exigiria um conjunto de providências, que levaria em conta as repercussões dessas medidas sobre o sistema de segurança e saúde psíquica 
dos trabalhadores. Prescreve-se para o AP a função de garantir a guarda das Unidades e a vigilância permanente do SP, no entanto, o efetivo cumprimento desta prescrição nem sempreé possível diante das condições reais de trabalho. As estratégi as adotadas pelos AP diante das adversidades podem, de alguma maneira, permitir a manutenção da funcionalidade das Unidades, mas podem também implicar importantes concessões quanto à natureza do trabal ho e incremento das cargas psíquicas.

Viu-se que as condições infraestruturais de trabalho, as dificuldades para a realização das atividades, jornada excessiva, outros aspectos relacionados à organização do trabalho (Fator 3) e um tempo maior no Sistema, também estavam associados a maiores prevalências de DPM. São, portanto, aspectos que também parecem contribuir para o desgaste da saúde mental dos agentes.

As maiores prevalências de DPM, associadas à indisponibilidade de tempo para o lazer e a ausência de prática de esporte, podem revelar a importância dessas atividades para o alívio das tensões existentes no ambiente desses trabalhadores.

Algumas variáveis não diretamente relacionadas ao trabal ho e que apareceram associadas ao estresse persistente foram: sexo feminino e ausência de prática de esporte. É possível que as mulheres, por razões culturais, sejam mais vulneráveis às tensões do trabalho em penitenciárias, e tenham menos capacidade de reagir a essas tensões. Nessas circunstâncias, a necessidade imperiosa de manter sua autoridade, deve ser altamente desgastante para elas. Deve-se acrescentar também, o possível efeito negativo representado pelo acúmulo com a jornada de trabalho doméstico (Santana, 1993). Viu-se que a prática de esporte e a disponibilidade de tempo para lazer, apareceram novamente minimizando o estresse apresentado.

Alguns aspectos relativos ao trabal ho do AP estavam estatisticamente associados com prevalências mais elevadas de estresse persistente: não realização de treinamento, mais de 48 horas semanais de trabal ho e exposição a um ambiente psicologicamente insatisfatório (Fator 2). O papel das horas totais semanais de trabalho evidencia que a atividade extra, remunerada, nas horas de folga, pode ser prejudicial para os agentes, provocando mais estresse e as conseqüências conhecidas deste sobre a saúde (Lipp \& Malagris, 1995). A presença do Fator 2 no modelo final, indica também, que um ambiente psicologicamente adequado, boa relação com o chefe e os colegas, maior indepen- dência nas ativi dades e maior supervisão minimizam o estresse.

Duas condições relativas ao trabalho; exercício de outra atividade remunerada e ausência de treinamento, sustentaram-se no modelo final para avaliação de suspeita de alcoolismo. É possível que fatores não ocupacionais, não investigados no presente estudo, estejam relacionados ao uso abusivo de álcool.

As condições de trabalho, associadas com queixas gerais de saúde, revelaram que as condições infra-estruturais (Fator 1 ), o contexto psicossocial (Fator 2) e a organização do trabaIho (Fator 3) se associaram a maiores prevalências de queixas. Além dessas, se o agente costumava dobrar o turno ou se trabalhava no SP há mais de nove anos, essas prevalências também foram maiores, sugerindo que as condições de trabal ho poderiam estar relacionadas com problemas físicos, além daqueles relativos à saúde mental.

Variáveis não ocupacionais, tais como aquelas relativas ao ambiente familiar, às condições nas quais cada trabalhador viveu antes do trabalho na penitenciária, e que poderiam influenciar sua saúde física e/ ou mental, não foram investigadas. Esse controle de possíveis variáveis confundidoras, relativas à vida extratrabalho, tem sido habitualmente negligenciado nas pesquisas da área Saúde e Trabalho (J Breilh, 2001).

A utilização de informações baseadas na opinião dos trabalhadores, procedimento muito comum em pesquisas epidemiológicas, especialmente aquelas de caráter exploratório, podem provocar superestimativas das exposições estudadas.

Os achados poderiam também estar distorcidos pelo efeito do trabalhador sadio. Contudo, não se pretende generalizar os resultados para a população geral. Os achados deste estudo são válidos para a categoria de trabalhadores investigada, já que foram realizadas apenas comparações internas ao grupo estudado.

Investigaram-se eventos de saúde de natureza crônica, não fatais, cujas medidas terapêuticas não oferecem habitualmente cura completa sem recidivas, o que parece mais adequado para o estudo do tipo transversal, a fim de minimizar o viés de sobrevivência determinado pela perda de óbitos ou curados.

Quanto ao viés de sobrevivência do trabaIhador sadio, que poderia resultar de demissões voluntárias ou involuntárias, de aposentadorias, transferências de função de trabalhadores doentes, além dos afastamentos por licença médica, algumas estratégias do estudo e características da população estudada pare- 
cem ter permitido minimizar este problema. Apenas $11,0 \%$ dos AP estavam desviados de função, e a análise realizada sem eles chegou a resultados semel hantes àqueles obtidos para todo o grupo estudado, o que pode sugerir que os deslocamentos não ocorreram devido à morbidade. Além disso, todos os AP exerciam empregos públicos, o que, no Brasil, ainda representa um grande grau de estabilidade. A população-alvo do estudo incluiu todos os trabalhadores e não apenas aqueles que se encontravam em atividade. Assim, no caso de licenças médicas, investigou-se o motivo do afastamento e decidiu-se por excluir do estudo apenas os trabalhadores cuja ausência não tivesse qualquer relação presumível com o trabalho.

Deve-se destacar que possíveis incorreções de classificação podem ter impedido o controle adequado de confundimento para algumas variáveis. Assim, apesar do ajuste obtido na análise multivariável, é possível haver confundimento residual não controlado no presente estudo. Na etapa de análise multivariável, muitas variáveis tiveram suas categorias reagrupa- das, o que pode ter resultado também em viés de classificação incorreta. No entanto, esse reagrupamento de categorias é inevitável para viabilizar-se etapas mais avançadas de análise estatística em quase todos os estudos epidemiológicos.

Pode-se considerar que a investigação apresenta uma validade interna aceitável, pois, apesar da possível ocorrência de viéses, estes não parecem ter sido de grande magnitude, tendo decorrido principalmente das limitações atuais do próprio método epidemiológico.

A validade externa do estudo pode também ser considerada boa, porque a definição do tamanho e a forma de seleção da amostra, utilizaram procedimentos adequados para se obter representatividade da população-alvo. Além disso, foram utilizados métodos estatísticos adequados para fazer-se inferência estatística. Contudo, deve ser lembrada a natureza exploratória deste estudo, sendo sua principal contribuição, a geração de hipóteses a serem testadas em outros trabalhos.

\section{Referências}

ARAÚJO, T. M.; SILVANY NETO, A. M .; REIS, E. J. F. B. \& KAVALKIEVICZ, C., 1998. Condições de TrabaIho e Saúde dos Professores da Rede Particular de Ensino de Salvador, Bahia. Relatório Técnico de Pesquisa. Salvador: Departamento de Medicina Preventiva, Faculdade de Medicina, Universidade Federal da Bahia/Departamento de Saúde, Sindicato de Professores no Estado da Bahia.

BERNARDES, E., 1997. À beira de um ataque de nervos. Veja, 1484:92-99.

BORGES, C. H., 1990. Transtornos Mentais Menores entre Trabal hadores de uma Usina Siderúrgica. Dissertação de Mestrado, São Paulo: Faculdade de Medicina, Universidade de São Paulo.

BRASI L, 1997. Sistema Penitenciário: Resumo do Quadro Indicador do Censo. Brasília: Conselho Nacional de Política Criminal e Penitenciária, Ministério da Justiça.

BREILH, J., 2001. Eficácia de poder, retroceso del derecho y degradación del trabajo. El escenario regresivo de la salud laboral en América Latina. In: Encontro Nacional de Saúde do Trabal hador, Anais, p. 196. Brasília: Departamento de Ações e Estratégicas, Secretaria de Políticas de Saúde, Ministério da Saúde. 
DANIEL, W. W., 1987. Bioestatistics: A Fundation for Analysis in the Health Sciences. 5 th Ed. New York: John Wiley \& Sons.

DEJOURS, C., 1987. A Loucura do Trabalho: Estudo de Psicopatologia do Trabalho. São Paulo: Editora Cortez.

FERNANDES, S. R. O., 1993. Trabalho Informatizado e Distúrbios Psicoemocionais: Estudo Seccional em Três Empresas de Processamento de Dados de Salvador-Bahia. Dissertação de Mestrado, Salvador: Faculdade de Medicina, Universidade Federal da Bahia.

GOLDBERG, P.; SIMONE, D.; LANDRE, M. F.; GOLBERG, M.; DASSA, S. \& FUHRER, R., 1996. Work condition and mental health among prison staff in France. Scandinavian Journal of Work and Environmental Health, 22:45-54.

HOSMER Jr., D. W. \& LEMESHOW, S., 1989. Applied Logistic Regression. New York: John Wiley \& Sons.

KLEINBAUM, D. G.; KUPPER, L. L. \& MULLER, K. E., 1988. Applied Regression Analysis and other Multivariable Methods. Boston: PWS-KENT.

LIPP, M. N. \& HOYOS, A. G., 1994. Validação empírica do inventário de sintomas de stress (ISS). Estudos dePsicologia, 11:43-49.

LIPP, M. N. \& MALAGRIS, L. N., 1995. Manejo do estresse. In: Psi coterapia Comportamental e Cognitiva: Pesquisa, Prática, Aplicações e Problemas (R. Bernard, org.), pp. 279-292, São Paulo: Editorial Psyll.

MARI, J., 1986. Minor Psychiatric Morbidity in Three Primary Medical Care Clinics in the City of São Paulo. Ph. D. Thesis, London: Institute of Psychiatry, University of London.

MASUR, J. \& MONTEIRO, M. G., 1983. Validation of the "cage" alcoholism screening test in a Brazilian psychiatric impatient hospital setting. Revista Brasileira de Pesqui sas Médicas e Biológicas, 16: 215-218.
OLIVEIRA, N. F.; SANTANA, V. S. \& LOPES, A. A., 1997. Razões de prevalência e uso do método delta para intervalos de confiança em regressão logística. Revista de Saúde Pública, 31:90-99.

PITTA, A., 1990. Hospital, Dor e Morte como Ofício. São Paulo: Editora Hucitec.

SANTANA, V. S., 1993. Informal Work, Housework and Minor Psychological Disorders among Women in Urban Brazil. Ph.D. Thesis, Chappel Hill: School of Public Health, University of North Carolina.

SILVA, E. S., 1993. Uma história de crise de nervos: Saúde mental e trabalho. In: Isto é Trabal ho de Gente?: Vida, Doença e Trabalho no Brasil (L. E. Rocha, R. M. Rigotto \& J. T. B. Buschinelli, org.), pp. 609-635, São Paulo: Editora Vozes.

STEENLAND, K.; LEVINE, A. J.; SIEBER, K.; SCHULTE, P. \& AZIZ, D., 1997. Incidence of tuberculosis infection among New York State Prison employees. American Journal of Public Health, 87:2012-2014.

SVENSON, L. W.; JARVIS, G. K.; CAMPBELL, R. L.; HOLDEN, R. W.; BACKS, B. J. \& LAGACE, D. R., 1995. Past and current drug use among Canadian correctional officers. Psychological Reports, 76: 977-978.

TARTAGLINI, A. J. \& SAFRAN, D. A., 1997. A topography of psychiatric disorders among correction officers. Journal of Occupational and Environmental Medicine, 39:569-573.

WISNER, A., 1994. A Inteligência no Trabal ho: Textos Sel ecionados de Ergonomia. São Paulo: Fundação Jorge Duprat Figueiredo de Segurança e Medicina do Trabalho.

Recebido em 22 de fevereiro de 2001

Versão final reapresentada em 17 de novembro de 2001 Aprovado em 17 de janeiro de 2002 